\title{
Prevalencia del Síndrome de Burnout en profesionales de enfermería de la Orinoquia colombiana, 2016
}

\author{
Prevalence of Burnout syndrome in nursing professionals from Orinoquia, Colombia in 2016
}

\author{
Oscar Alexander Gutiérrez-Lesmes' ${ }^{1}$ orcid.org/0000-0002-5181-0236 \\ Nelly Johana Loboa-Rodríguez ${ }^{*}$ orcid.org/0000-0001-8999-5072 \\ Javier Martínez-Torres ${ }^{1}$ orcid.org/0000-0001-8991-5079
}

1 Universidad de los Llanos. Villavicencio, Colombia

Fecha de recepción: Mayo 4-2017 Fecha de revisión: Noviembre 10-2017 Fecha de aceptación: Diciembre 21 - 2017

Gutiérrez-Lesmes OA, Loboa-Rodríguez NJ, Martínez-Torres J. Prevalencia del Síndrome de Burnout en profesionales de enfermería de la Orinoquia colombiana, 2016. Univ. Salud. 2018;20(1):37-43. DOI: http://dx.doi.org/10.22267/rus.182001.107

\section{Resumen}

Introducción: El síndrome de Burnout es descrito como una inadecuada forma de afrontar el estrés laboral, los rasgos principales son el agotamiento emocional, la despersonalización y disminución del desempeño personal. Objetivo: Determinar la prevalencia del Síndrome de Burnout en los profesionales de enfermería que laboran en la Orinoquia colombiana. Materiales y métodos: Se realizó un estudio analítico transversal, muestreo por intención, la población de estudio estuvo constituida por 100 enfermeros, se utilizó el instrumento de Maslach Burnout. Se calcularon medidas de resumen, prevalencia y razón de prevalencia, con el programa SPSS. Resultados: El 90\% de la muestra estudiada eran mujeres, 53\% solteros, 54\% menores de 28 años y $42 \%$ sin hijos. La prevalencia general del síndrome de Burnout fue de 16\%, en el área de atención hospitalaria fue de un 25,5\% y en áreas diferentes a esta fue 6,1\%; en el área hospitalaria se presentaron mayores proporciones en cansancio emocional 42\%, despersonalización emocional 38\%, falta de realización personal 30\%, se encontró diferencias estadísticamente significativas para la prevalencia del síndrome de Burnout en el área de atención hospitalaria (RP 4,2, IC95\% 1,3-13,7, P 0,008), para profesionales menores de 28 años (RP 3,7 IC95\% 1,1- 12,1, P 0,017). Conclusiones: La prevalencia del síndrome de Burnout es mayor en enfermeros del área hospitalaria, tener menos de 28 años se asoció a una mayor prevalencia, los enfermeros clasificados como enfermos con síndrome de Burnout debían cuidar un mayor número de pacientes en su jornada laboral.

Palabras clave: Agotamiento profesional; enfermería; atención hospitalaria; prevalencia; estudios transversales. (Fuente: DeCS, Bireme).

\begin{abstract}
Introduction: Burnout syndrome is described as an inadequate way of coping with labor stress. The main traits are emotional exhaustion, depersonalization and decreased personal performance. Objective: To determine the prevalence of Burnout syndrome in nursing professionals working in the Colombian Orinoquia. Materials and methods: A transverse analytical study was carried out with sampling by intention. The study population was constituted by 100 nurses and the Maslach Burnout instrument was used. Summary, prevalence and prevalence ratio measurements were calculated with the SPSS program. Results: $90 \%$ of the sample studied were women, 53\% single, $54 \%$ under 28 and $42 \%$ without children. The general prevalence of Burnout syndrome was $16 \%$, in the area of hospital care was $25.5 \%$ and in areas other than this was $6.1 \%$. In the hospital area, there were greater proportions in emotional fatigue with a $42 \%$, emotional depersonalization with a $38 \%$ and lack of personal realization with a $30 \%$. Statistically significant differences for the prevalence of Burnout syndrome in the area of hospital care were found (RP
\end{abstract}


4.2, IC95\% 1,3-13.7, P 0.008) for professionals under 28 (RP 3.7 IC95\% 1.1-12.1, p 0.017). Conclusions: The prevalence of Burnout syndrome is higher in nurses in the hospital area. Being under 28 years of age was associated with a higher prevalence. Nurses classified as sick with Burnout syndrome had to take care of a greater number of patients in their workday.

Keywords: Burnout, professional; nursing; hospital care; prevalence; cross-sectional studies. (Source: DeCS, Bireme).

\section{Introducción}

El síndrome de Burnout, también conocido como síndrome de desgaste profesional, de sobrecarga emocional o de fatiga en el trabajo, fue declarado en el año 2000 por la Organización Mundial de la Salud (OMS) como un factor de riesgo laboral(1). Este se ha estudiado principalmente bajo la definición de Maslach y Jackson, quienes lo describen como una forma inadecuada de afrontar el estrés crónico, sus rasgos principales son el agotamiento emocional, la despersonalización y la disminución del desempeño personal(2-6), otra definición es la propuesta por Gil(7) quien lo define desde la perspectiva psicosocial, como una respuesta al estrés laboral crónico caracterizado por una percepción de fracaso por parte de quien lo padece. Se presenta con mayor frecuencia entre aquellas ocupaciones que tienen contacto directo y constante con otras personas $(8)$, factores de riesgo ocupacional como condiciones de tensión, la labor en sus contenidos y niveles de responsabilidad, carga emocional en cada $\operatorname{rol}^{(4,9) \text {. }}$

Existe consenso entre algunos autores, que definen el contacto con personas o pacientes y su complejidad como causa de la aparición del síndrome, de ahí que el personal de enfermería presente comportamientos particulares en la prevalencia de síndrome de Burnout, dependiendo del tipo de servicio o área donde labore( ${ }^{(4,8,10-13)}$.

El objetivo del presente estudio fue establecer la prevalencia del síndrome de Burnout en los profesionales de enfermería que laboran en la Orinoquia colombiana, comparando aquellos que trabajan en atención hospitalaria con quienes trabajan en áreas diferentes a la hospitalaria.

\section{Materiales y métodos}

\section{Tipo de estudio y población}

Se realizó un estudio analítico transversal, el cual se llevó a cabo en profesionales de enfermería que ejercen su labor en la Orinoquia colombiana (Arauca, Casanare, Meta y Vichada). Se tomó una muestra de 100 sujetos, la cual fue de carácter no probabilística con muestreo por intención; los enfermeros se tomaron de los siguientes lugares de trabajo: diez de centros hospitalarios de segundo y tercer nivel, ocho de centros de atención primaria, seis de Secretarías Locales de Salud y tres de entidades del estado.

\section{Proceso de medición y recolección}

El procedimiento para la recolección de información fue mediante la aplicación de entrevista a los participantes en cada ciudad de residencia. El instrumento utilizado fue Burnout Maslach Inventory validado en Colombia en el 2004, por Chávez y Castrillón quienes obtuvieron una consistencia interna de 0,62 para agotamiento emocional, 0,72 para despersonalización y 0,76 para realización personal(14,15), los criterios de inclusión fueron ser profesional de enfermería, que labore actualmente en el área clínica o de salud pública en la Orinoquia colombiana (Arauca, Casanare, Meta y Vichada) y que decida participar de manera voluntaria en el estudio. Los criterios exclusión fueron: que al momento del estudio trabajara de manera simultánea en el área de salud pública y clínica.

\section{Análisis estadístico}

Se realizó un análisis exploratorio para las variables cuantitativas, para determinar su distribución (valores extremos, simetrías, curtosis, tipos de distribución); para las variables cualitativas se analizaron frecuencias relativas (moda, datos erróneos y datos perdidos). 
Posteriormente, se analizó mediante estadística descriptiva, análisis univariado (proporciones, tendencia central y dispersión) y análisis bivariado, para lo cual se calculó razón de prevalencia, intervalo de confianza y significancia estadística (las variables se categorizaron usando la mediana como punto de corte). Para las variables numéricas se compararon las medias, la comparación de las variables cuantitativas se llevó por medio de t-student, para muestra independientes con varianzas iguales 0 desiguales, según sea el caso, (se evaluaó la normalidad con la prueba de Shapiro-Wilk e independencia). El análisis se realizó en SPSS, se consideró como significativo un valor $p<0,05$. Para la estimación de parámetros se usaron intervalos de confianza del 95\%.

\section{Consideraciones éticas}

El estudio fue revisado y aprobado por el Comité de Ética de la Universidad de los Llanos, de acuerdo a la Resolución 8430 de 1993(16) de la normatividad colombiana y a la Declaración de Helsinki. Este estudio se consideró de riesgo ético mínimo, se aplicó un formato de consentimiento informado previo a la obtención de la información, la participación fue voluntaria.

\section{Resultados}

Las características sociodemográficas de los participantes se distribuyen así: El 90\% pertenecen al sexo femenino; el $53 \%$ son solteros, el $22 \%$ casado y el $20 \%$ se encuentran en unión libre; el 54\% tiene menos de 28 años; el $42 \%$ aún no tiene hijos, el 59\% tienen menos de 6 años de experiencia laboral, $66 \%$ trabaja 8 horas o más al día, $65 \%$ brinda cuidado a 11 o más pacientes al día, el $27 \%$ posee simultáneamente dos empleos, el $85 \%$ tiene contratación laboral directa, 61\% trabaja en una institución pública (Tabla 1).

Los enfermeros encuestados de la Orinoquia, mostraron una prevalencia síndrome de Burnout del 16\%; la distribución según el área de trabajo evidenció que laborar en atención hospitalaria genera una prevalencia del $25,5 \%$, mientras que los enfermeros que trabajan en áreas diferentes presentan una prevalencia de 6,1\%. El 42\% de los enfermeros que laboran en atención hospitalaria presentaron un alto cansancio emocional, el 38\% una alta despersonalización y 30\% elevada falta de realización personal. Los enfermeros de otras áreas presentan proporciones más bajas en las tres dimensiones: cansancio emocional (20\%), alta despersonalización (26\%) y falta de realización personal (24\%) (Tabla 2).

Tabla 1. Variables sociodemográficas y relacionadas con el ámbito laboral en profesionales de enfermería Orinoquia

\begin{tabular}{|c|c|c|}
\hline & $n$ & $\%$ \\
\hline \multicolumn{3}{|l|}{ Sexo } \\
\hline Hombre & 10 & 10 \\
\hline Mujer & 90 & 90 \\
\hline \multicolumn{3}{|l|}{ Estado civil } \\
\hline Soltero & 53 & 53 \\
\hline Casado & 22 & 22 \\
\hline Separado & 4 & 4 \\
\hline Unión libre & 20 & 20 \\
\hline Otros & 1 & 1 \\
\hline \multicolumn{3}{|l|}{ Hijos } \\
\hline No & 42 & 42 \\
\hline $\mathrm{Si}$ & 58 & 58 \\
\hline \multicolumn{3}{|l|}{ Edad } \\
\hline Menor a 28 años & 54 & 54 \\
\hline Mayor a 28 años & 46 & 46 \\
\hline \multicolumn{3}{|c|}{ Años de experiencia laboral } \\
\hline Menor a 6 años & 59 & 59 \\
\hline Mayor a 6 años & 41 & 41 \\
\hline \multicolumn{3}{|c|}{ Número de empleos actuales } \\
\hline Un empleo & 73 & 73 \\
\hline Dos empleos & 27 & 27 \\
\hline \multicolumn{3}{|c|}{ Horas que trabaja al día } \\
\hline Menor a 8 horas & 34 & 34 \\
\hline Mayor a 8 horas & 66 & 66 \\
\hline \multicolumn{3}{|c|}{ Pacientes que asiste en una jornada laboral } \\
\hline Menor a 11 & 33 & 35 \\
\hline Mayor a 11 & 66 & 65 \\
\hline \multicolumn{3}{|c|}{ Tipo de contratación } \\
\hline Directa & 85 & 85 \\
\hline Cooperativa & 15 & 15 \\
\hline \multicolumn{3}{|c|}{$\begin{array}{l}\text { Naturaleza de la institución en la que trabaja } \\
\text { actualmente }\end{array}$} \\
\hline Pública & 61 & 61 \\
\hline Privada & 39 & 39 \\
\hline
\end{tabular}


Tabla 2. Proporción, dimensiones cansancio emocional, despersonalización y falta de realización según área de atención

\begin{tabular}{|c|c|c|c|c|c|c|c|c|c|}
\hline & \multirow[t]{2}{*}{$n$} & \multicolumn{2}{|c|}{$\begin{array}{c}\text { Cansancio } \\
\text { emocional \% }\end{array}$} & \multicolumn{2}{|c|}{$\begin{array}{c}\text { Despersonalización } \\
\%\end{array}$} & \multicolumn{2}{|c|}{$\begin{array}{c}\text { Falta de } \\
\text { realización } \\
\text { personal }\end{array}$} & \multirow[t]{2}{*}{$\begin{array}{l}\text { Prevalencia } \\
\text { Burnout por } \\
\quad \text { área \% }\end{array}$} & \multirow[t]{2}{*}{$\begin{array}{l}\text { Prevalencia } \\
\text { Burnout } \\
\text { global \% }\end{array}$} \\
\hline & & Bajo & Alto & Baja & Alta & Baja & Alta & & \\
\hline \multirow{2}{*}{$\begin{array}{l}\text { Atención hospitalaria } \\
\text { Áreas diferentes a la } \\
\text { atención hospitalaria }\end{array}$} & 51 & 58 & 42 & 62 & 38 & 70 & 30 & 25,5 & \multirow[b]{2}{*}{16,0} \\
\hline & 49 & 80 & 20 & 74 & 26 & 76 & 24 & 6,0 & \\
\hline
\end{tabular}

Los resultados hallados en el cálculo de razón de prevalencia para las distintas variables presentaron diferencias estadísticamente significativas para el área de trabajo y la edad de los participantes. Se encontró asociación estadística entre laborar en atención hospitalaria asistencial (RP 4,2 IC95\% 1,3-13,7 p=0,008), y ser menor de 28 años (RP 3,7 IC95\% 1,1-12,1 $\mathrm{p}=0,017$ ) (Tabla 3).

Tabla 3. Razón de prevalencia del síndrome de Burnout

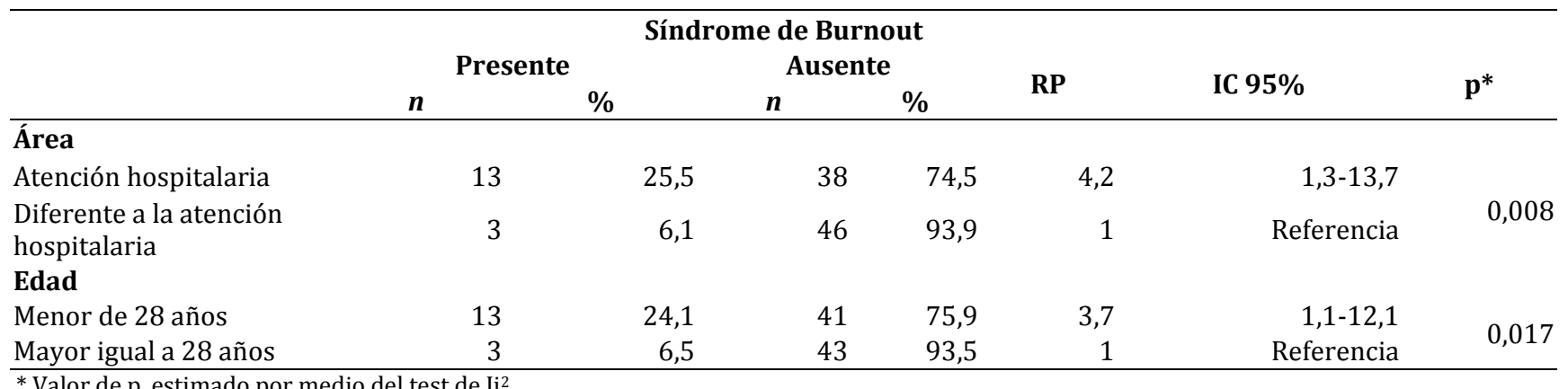

* Valor de p, estimado por medio del test de $\mathrm{ji}^{2}$.

Los profesionales con síndrome de Burnout presentan un promedio más alto para las variables: horas que trabaja al día, horas que trabajará en el mes, y un número de pacientes que asiste en una jornada laboral, para el número de pacientes se encontró diferencia en la comparación de medias (Tabla 4).

Tabla 4. Comparación de carga laboral de los enfermeros, clasificados según prevalencia del síndrome Burnout

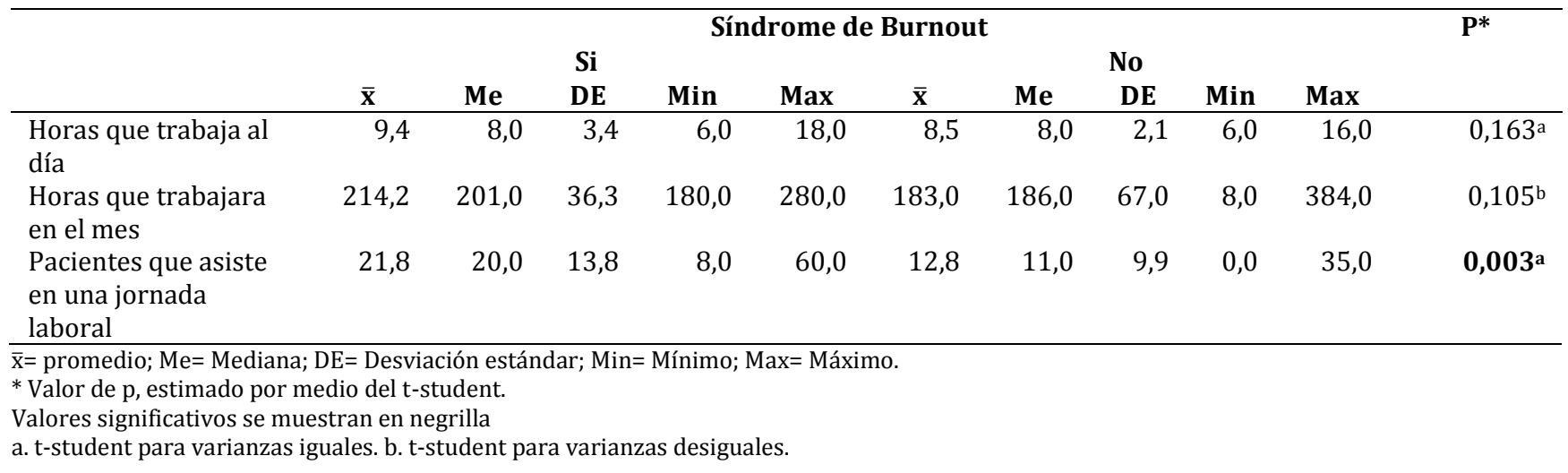




\section{Discusión}

Condiciones del ambiente hospitalario y las características de la funciones de enfermería han hecho que esta sea catalogada como una profesión de riesgo y mayor prevalencia para el desarrollo del síndrome de Burnout(5,17-19). En Colombia se han desarrollado investigaciones (en ciudades especificas), que se han orientado a describir la prevalencia del síndrome o sus factores asociados, se han realizado principalmente en docentes $(20,21)$ y en profesionales de la salud(14,15,22-25).

La prevalencia global del síndrome de Burnout para la muestra tomada fue del $16 \%$, en los profesionales que laboran en el área hospitalaria fue de 25,5\%, presentaron una prevalencia superior en un $19 \%$ con respecto a aquellos que laboran en otras áreas.

La diferencia entre los que laboran en el área de atención hospitalaria, frente a los que trabajan en un área diferente, se explica por las características propias del trabajo hospitalario de enfermería: una mayor carga laboral (número de pacientes y tiempo de trabajo)(19,24,26-28), carga emocional (exposición al dolor, la muerte) junto con la responsabilidad sobre el bienestar del paciente ${ }^{(5,19)}$ y la rutina $\operatorname{diaria}^{(18,19)}$. La menor prevalencia del síndrome en enfermeros que laboran en áreas no hospitalarias, también fue encontrada en un estudio realizado en Suiza, donde asocian este hallazgo a la autonomía laboral que se presenta en esta área(29).

En Colombia se ha reportado diferentes prevalencias en profesionales de enfermería que laboran en el área de atención hospitalaria: 15,5\% en Barranquilla(23), 26,6\% en Cartagena (24), 24,4\% en Ibagué(30), 17,5\% en Bogotá(31), 9,1\% en Tunja(26), resultados que concuerdan con los hallados en el presente estudio, ubicando la prevalencia global de Burnout y la prevalencia de los profesionales que trabajan en el área de atención hospitalaria en la Orinoquia dentro de estos valores, pero más cerca de la prevalencia encontrada en Ibagué y Cartagena. El hallazgo de la asociación en mayores de 28 años ya fue descrito de igual manera en Cartagena y
Boyacá(24,32), aunque en los estudios referenciados para Barranquilla(23) e Ibagué(30) no se encontró ninguna relación y el realizado en Bogotá(31) encontró que a mayor edad mayor prevalencia. La relación de la edad con la prevalencia encontrada en la Orinoquia es explicada por otros estudios que argumentan que existe una relación inversa entre la edad y este síndrome (33), posiblemente asociada al afrontamiento eficaz con el tiempo(32). En el resto de variables medidas en este estudio no se encontró significancia estadística para las asociaciones $(>0,05)$.

El comportamiento de las variables sociodemográficas y laborales es similar a lo descrito por otros autores; en el presente estudio, el $90 \%$ de los encuestados pertenece al sexo femenino, como se ha encontrado en diferentes estudios $(5,17,23,24,26,27,30,31,34,35)$, desde 1860, cuando Florence Nigthingale direcciona a la enfermería a un nivel de profesión, hasta la actualidad, la función de las enfermeras en las instituciones de salud obedece al estereotipo del género femenino(36). El estado civil predominante es ser solteros, seguido por estar casado, igual que lo encontrado en Ibagué(30), pero en Bogotá(31), Barranquilla(23) y Cartagena(24) esta condición es minoritaria, aunque el estudio realizado en la Orinoquia no encontró relación de la variable estado civil con la ocurrencia del síndrome, sin embargo, este ha sido asociado en un estudio realizado en Grecia(18). El 58\% de los enfermeros tenían al menos un hijo, cuando se exploró la razón de prevalencia para esta variable no se encontró asociación con la presencia del síndrome de Burnout, caso contrario a lo encontrado en los estudios realizados en Bogotá(31), Cartagena(24) e Ibagué(30) donde encontraron relación entre tener hijos y la prevalencia del síndrome de Burnout.

Los enfermeros con síndrome de Burnout presentan un promedio más alto de horas de trabajo al día, y horas que trabaja en al mes, comparados con los que no fueron clasificados con este síndrome. En cuanto al número de pacientes que asiste en una jornada laboral, se encontró diferencia en la comparación de medias, estos hallazgos sobre el número de pacientes 
como factor relacionado con la prevalencia son descritos en los estudios ya mencionados en diferentes ciudades de Colombia.

\section{Conclusiones}

Los profesionales de enfermería en la Orinoquia padecen del síndrome de Burnout, con mayor prevalencia en aquellos que laboran en al área de atención hospitalaria comparados con aquellos que trabajan en áreas diferentes y en los menores de 28 años, los enfermeros clasificados como positivos para el síndrome son los que atienden mayor número de pacientes en su jornada laboral.

\section{Fuente de financiación}

El proyecto fue financiado por la Universidad de los Llanos para la ejecución del protocolo, código C05-F03-010-2016, Proyecto VIAC-6823112015Fomento a la investigación.

\section{Agradecimientos}

A los enfermeros César Torres Muñoz y Oscar Alirio Torres por su apoyo en la ejecución del proyecto.

\section{Conflicto de intereses}

Los autores declaran que no existen conflictos de intereses.

\section{Referencias}

1. Saborío L, Hidalgo LF. Síndrome de Burnout. Medicina Legal de Costa Rica. 2015;32(1):119-6.

2. Durán M, Rodríguez R, Fernández L. Prevalencia del síndrome del quemad@y estudio de factores relacionados en 1@s enfermer@s del chuvi (complexo hospitalario universitario de vigo). Enfermería Global. 2006;5(1):1-18.

3. Martín M, Fernández F, Gómez R, Martínez F. Prevalencia y factores asociados al burnout en un área de salud. Aten Primaria. 2001;27(05):313-7.

4. Ordenes N. Prevalencia de Burnout en trabajadores del hospital Roberto del Río. Revista chilena de pediatría. 2004;75(5):449-6.

5. Wang S, Liu Y, Wang L. Nurse burnout: Personal and environmental factors as predictors. International journal of nursing practice. 2015;21(1):78-86.

6. Salvagioni D, Melanda F, Mesas A, González A, Gabani F, de Andrade S. Physical, psychological and occupational consequences of job burnout: A systematic review of prospective studies. PloS one. 2017;12(10):1-29.

7. Gil-Monte P. Perspectivas teóricas y modelos interpretativos para el estudio del síndrome de quemarse por el trabajo. Anales de psicología. 1999;15(2):261-8.

8. Albaladejo R, Villanueva R, Ortega P, Astasio P, Calle M, Domínguez V. Síndrome de Burnout en el personal de enfermería de un hospital de Madrid. Revista española de salud pública. 2004;78(4):505-12.

9. Tabla de Enfermedades Laborales, Decreto 147705 de Agosto 2014. Sect. Diario Oficial No 49234 (2014).

10. López-Soriano F, Bernal L. Prevalencia y factores asociados con el síndrome de burnout en enfermería de atención hospitalaria. Revista de Calidad asistencial. 2002;17(4):201-5.

11. Vidal P, Ferrer A. Síndrome de Burnout en profesionales de enfermería de cuidados críticos. Enfermería Intensiva. 2002;13(1):9-18.

12. Linde J, Martínez F, Cervantes I. Burnout en enfermería de atención hospitalaria. Enfermería clínica. 2005;15(5):275-8.

13. Atance J. Aspectos epidemiológicos del síndrome de burnout en personal sanitario. Revista española de salud pública. 1997;71(3):293-11.

14. Restrepo-Ayala N, Colorado-Vargas G, Cabrera-Arana G. Desgaste emocional en docentes oficiales de Medellín, Colombia, 2005. Revista de Salud Pública. 2006;8(1):6311.

15. Rojas ML, Zapata JA, Grisales H. Síndrome de Burnout y satisfacción laboral en docentes de una institución de educación superior, Medellín, 2008. Revista Facultad Nacional de Salud Pública. 2009;27(2):198-10.

16. Facultad Nacional de Salud Pública. Morbilidad y mortalidad de la población colombiana Bogotá (CO): Imprenta Nacional de Colombia;2011.

17. Copanitsanou P, Fotos N, Brokalaki H. Effects of work environment on patient and nurse outcomes. British Journal of Nursing. 2017;26(3):172-6.

18. Lahana E, Papadopoulou K, Roumeliotou O, Tsounis A, Sarafis P, Niakas D. Burnout among nurses working in social welfare centers for the disabled. BMC nursing. 2017;16(1):1-15.

19. Khamisa N, Peltzer K, Oldenburg B. Burnout in relation to specific contributing factors and health outcomes among nurses: a systematic review. International journal of environmental research and public health. 2013;10(6):2214-27.

20. Rodríguez V, Dávila M, Avella-García C, Caballero A, Vives N, Mora C, et al. Prevalencia y características del síndrome de agotamiento profesional (SAP) en docentes de tres colegios públicos de Bogotá (Colombia). Revista colombiana de psiquiatría. 2009;38(1):50-16.

21. Correa-Correa Z, Muñoz-Zambrano I, Chaparro A. Síndrome de Burnout en docentes de dos universidades de Popayán, Colombia. Revista de salud pública. 2010;12(4):589-10.

22. Paredes O, Sanabria-Ferrand P. Prevalencia del síndrome de Burnout en residentes de especialidades médico quirúrgicas, su relación con el bienestar psicológico y con variables sociodemográficas y laborales. Revista Médica. 2008;16(1):25-8.

23. Tuesca-Molina $R$, Iguarán $M$, Suárez $M$, Vargas $G$, Vergara D. Síndrome de desgaste profesional en 
enfermeras/os del área metropolitana de Barranquilla. Salud Uninorte. 2006;22(2):84-8.

24. Cogollo-Milanés Z, Batista E, Cantillo C, Jaramillo A, Rodelo D, Meriño G. Desgaste profesional y factores asociados en personal de enfermería de servicios de urgencias de Cartagena, Colombia. Aquichan. 2010;10(1):43-9.

25. Guevara C, Henao D, Herrera J. Síndrome de desgaste profesional en médicos internos y residentes. Hospital Universitario del Valle, Cali, 2002. Colomb med. 2004;35(4):173-8.

26. Álvarez L, Bocanegra P, Migdolia B. Prevalencia de desgaste profesional en personal de enfermería de un hospital de tercer nivel de Boyacá, Colombia. Enfermería global. 2013;12(29):73-15.

27. Nie Z, Jin Y, He L, Chen Y, Ren X, Yu J, et al. Correlation of Burnout with social support in hospital nurses. International journal of clinical and experimental medicine. 2015;8(10):19144-6.

28. van der Doef M, Mbazzi FB, Verhoeven C. Job conditions, job satisfaction, somatic complaints and Burnout among East African nurses. Journal of Clinical Nursing. 2012;21(11-12):1763-13.

29. Heeb J-L, Haberey-Knuessi V. Health professionals facing Burnout: what do we know about nursing managers? Nursing research and practice. 2014;2014.

30. Grisales-Romero H, Muñoz Y, Osorio D, Robles E. Síndrome de Burnout en el personal de enfermería de un hospital de referencia Ibagué, Colombia, 2014. Enfermería Global. 2016;15(41):244-14.

31. Muñoz A, Velásquez M. Síndrome de quemarse por el trabajo en profesionales de enfermería, Bogotá, Colombia. Facultad Nacional de Salud Pública. 2016;34(2):202-9.

32. Bocanegra P, Migdolia B, Álvarez Verdugo LP. Prevalencia de desgaste profesional en personal de enfermería de un hospital de tercer nivel de Boyacá, Colombia. Enfermería global. 2013;12(1):73-16.

33. Jiménez B, Gutiérrez J, Hernández E. Variables sociodemográficas en el proceso de desgaste profesional de enfermería. Revista de ROL de Enfermería. 2002;25(11):18-9.

34. Gutiérrez-Lesmes 0 . Factores que determinan la aplicación del proceso de enfermería en instituciones hospitalarias de Villavicencio, Colombia, 2008. Avances en Enfermería. 2009;27(1):60-9.

35. Cogollo Z, Gómez E. Condiciones laborales en enfermeras de Cartagena, Colombia. Avances en enfermería. 2010;28(1):31-8.

36. García, Sainz A, Botella M. La enfermería vista desde el género. Index de Enfermería. 2004;13(46):45-4. 